\title{
An unusual collection: Nasal septal abscess secondary to a Furuncle
}

\section{Heeral Bhaga}

University of the Witwatersrand

Shivesh Maharaj ( $\nabla$ shivesh.maharaj@wits.ac.za )

University of the Witwatersrand https://orcid.org/0000-0002-2118-2400

\section{Short Report}

Keywords: nasal furuncle, nasal hematoma

Posted Date: July 27th, 2020

DOI: https://doi.org/10.21203/rs.3.rs-48164/v1

License: (c) (1) This work is licensed under a Creative Commons Attribution 4.0 International License. Read Full License 


\section{Abstract}

Nasal septal abscess is an uncommon entity and most likely to occur with nasal septal hematoma associated with trauma. It occurs when pus collects between the mucoperichondrium and the nasal septal cartilage. Complications can be avoided by prompt identification and initiation of appropriate treatment. Thus avoiding systemic and cosmetic sequelae.

We present a septal abscess secondary to nasal furuncle in a tertiary institute. This case report also serves to illustrate the investigations, recommended management and possible treatment outcomes.

\section{Case Presentation}

A nasal septal abscess is most commonly encountered secondary to a nasal hematoma which has been neglected. ${ }^{1,2}$ It is one the rarer complications associated with nasal furunculosis. ${ }^{3,4}$ We present this rare occurrence in a 73yr old hypertensive female. We have reviewed literature on nasal septal abscess, their causes, complications and principles of management.

We present a $73 \mathrm{yr}$ old female, Ms M. She was referred to our institute with a week history of bilateral nasal swelling and congestion. It was associated with anosmia and pain. She reported no rhinorrhea. Her congestion and pain were worsening in intensity.

She denied any history of trauma or foreign body insertion. She reported it as starting with a burning pain at a pimple which she then wiped with a tissue.

Her co-morbidities included hypertension and a previous history of right breast cancer which was treated with resection and adjuvant chemotherapy.

Anterior rhinoscopy revealed a mucosal covered purplish mass bilaterally obstructing the nasal cavity. There was minimal nasal misting. It was fluctuant on palpation. She has tenderness of the nasal dorsum. There was no cellulitis of surrounding tissue and no spread clinically beyond the nasal cavity. She was alert and maintained her airway via mouth breathing.

Her vital signs were stable throughout her admission and stay in hospital. She was afebrile. Her fasting glucose was $6.7 \mathrm{mmol}$. Her hypertension was well controlled and she showed no signs of immunocompromise.

She did not have any neurological fallout. Her blood work up showed a raised CRP of 36 and white cell count of 11.21 .

She was treated with an initial incision and drainage under local anaesthetic at the bedside. $7.5 \mathrm{ml}$ of pus was drained and packed bilaterally with BIPP. Ms M removed the BIPP before $48 \mathrm{hrs}$ as a result there was a recollection of fluid. The patient was then taken for a formal incision and drainage under general anaesthetic with repeat incision and drainage of septal hematoma. 
(Figure 1)

The anterior cartilaginous septum was found to be deficient. Juxtaposed quilting sutures were inserted. Her nose was packed bilaterally with merocell, which was removed the following day.

Ms M made a good recovery. Her antibiotic was changed to oral Cloxacillin which was culture directed to the Staphylococcus aureus that was cultured. She was discharged $48 \mathrm{hrs}$ post op when her septic markers decreased, CRP to 26 and White cell count of 9.

She returned a week later for a review. The sutures were in situ and she was found to have a septal deviation to the right and still complaining of nasal congestion. The sutures were removed Nasal douching was advised and she was booked for a follow up.

\section{Discussion}

A literature review was performed using PubMed and Google Scholar using the search terms nasal septal hematoma and septal abscess. It was not limited to date but to the English language.

Nasal septal abscesses secondary to a furuncle is a rare occurrence. It usually arises from a septal hematoma. A nasal septal abscess in the immunocompromised setting usually occurs secondary to insulin and non-insulin dependent diabetes, HIV, haematological malignancies and chemotherapy ${ }^{5}$.

Nwosu and Jones presented a prospective study detailing management and outcomes of septal hematomas /abscess. They found out of 53 patients in the study only 4 patients were secondary to furuncles. With the average presentation to a healthcare facility being 2 weeks. The major presenting symptom being nasal obstruction. All had successful outcomes with incision and drainage ${ }^{6}$.

Matsuba and colleagues presented an unusual case of septal abscess secondary to isolated sphenoid sinus information. The most common pathogen isolated in most studies was Staphylococcus aureus. This was on gram stain culture of the pus. ${ }^{4}$

The nasal septum is the main support structure of the nose. It is made up of cartilaginous and bony components. It is covered on either side by mucoperichondrium- mucoperiosteal membrane.

Blood supply to the nasal septum is derived from branches of the external and internal carotid artery. The septum has many submucosal vessels supplying it. The mucoperichondrium itself has various arteriovenous anastomosis. The cartilaginous nasal septum gets it blood supply via the mucosa covering it. The cartilage itself is avascular 7,8 .

Therefore any instance where there is a separation of the mucoperichondrium from the cartilage or expansion and obstruction of the blood vessels supplying the cartilage, it will result in compromise of its blood supply and progressive avascular necrosis within 3 days. If a hematoma does occur it is an ideal medium for colonization and growth of bacteria ${ }^{1}$. 
Nasal septal abscess is defined as a collection of purulent material between the cartilaginous or bony septum and the mucoperichondrium or mucoperiosteum ${ }^{2}$. It in itself is a rare occurrence. Other less common causes include iatrogenic, sphenoid or ethmoid sinusitis, dental abscess, nasal furuncle and tobacco sniffing (6). Contributing factors to septal abscess include retroviral disease, insulin dependent Diabetes Mellitus and sarcoidosis. The first reported case of nasal septal abscess was in 1810 by Cloquet. The rate of septal abscess post septoplasty reported as being between 0.4 to $12 \%^{3}$

Most common presentation of nasal septal abscess is with bilateral nasal congestion, septal and dorsal swelling and pain and low-grade fevers. Clinically a mucosal covered mass can be seen obstructing bilateral nasal cavities with tenderness to palpation.

The most common pathogen identified is Staph Aureus. Streptococcus and other anaerobes less commonly. In the paediatric population Haemophilus influenza is more common. Other rare pathogens that have been reported are Pseudomonas and Klebsiella. Fungal causes have also been described in immunocompromise individuals ${ }^{8}$.

To confirm diagnosis a CT Scan of nose and paranasal sinuses is recommended. The typical appearance of an abscess should be seen with a rim enhancing lesion between the mucoperichondrium/ mucoperiosteal and the cartilage. This finding can be missed on CT Brain ${ }^{2}$.

CT scanning recommended when there is facial/ periorbital cellulitis, significant headache, symptoms of meningitis, altered consciousness or localizing neurological signs. Failure to improve clinically post incision and drainage, delay in diagnosis ${ }^{7}$.

CT scan is also recommended especially if the underlying cause is uncertain. If the patient is suspected of having Wegener's granulomatosis, TB, Syphilis, Sarcoma or lymphoma especially in spontaneous onset of hematomas ${ }^{7}$.

Management of septal abscess requires prompt identification and surgical drainage, with intravenous or oral antibiotics. Most commonly a hemi-transfixtion incision is used. With $0.9 \%$ saline washout. Quilting sutures and nasal packing can be used to prevent re accumulation of pus/ blood. If at the time of incision and drainage marked destruction of the cartilage is noted a decision for reconstruction can be made 5 .

Complications of septal abscess include destruction of cartilaginous septum with resultant saddle nose deformity, intracranial complication including meningitis, cerebral abscess, subarachnoid empyema and cavernous sinus thrombosis. It may also result in functional disturbance of the nasal airflow ${ }^{6}$

\section{Declarations}


The present study was performed in accordance with the University of the Witwatersrand Human Research Ethics

protocol.

Patient consent was obtained for publication of this case report and related images

Consent for publication: All authors have consented to publication.

Availability of supporting data: all data are available on request to the corresponding,

Shivesh.maharaj@wits.ac.za

Competing interests: None to declare

Funding: The study is funded by the authors

Authors' contributions: SM and KH conceived the study and SM identified the data. SM extracted the data and undertook the analyses, assisted by $\mathrm{KH}$.SM prepared the initial draft the paper which both authors revised. The corresponding author attests that all listed authors meet authorship criteria and that no others meeting the criteria have been omitted.

Acknowledgements: None

\section{References}

1. Ambrus PS, Eavey RD, Baker AS, et al. Management of nasal septal abscess. Laryngoscope 1981;91: 575-82

2. Debnam J.M. Gillenwater A.M. Ginsberg L.E. Nasal Septal Abscess in Patients with Immunosuppression.J Neuroradiol. 2007 Nov-Dec 28:1878-79 [

3. Ketcham AS, Han JK. Complications and management of septoplasty. Otolaryngol Clin N Am. 2010;43:897-904. doi: 10.1016/j.otc.2010.04.013.

4. Matsuba HM, Thawley SE. Nasal septal abscess: unusual causes, complications, treatment and sequelae. Annals of plastic surgery. 1986 feb; 16(2): 161-166

5. Mooney C. P, Rimmer J. Spontaneous nasal septal hematoma and abscess: a case report and literature review. Rhinology online, Vol 1:122-126, 2018

6. Nwosu , J. N, Nnadede. Nasal septal hematoma/ abscess: management and outcome in a tertiary hospital of a developing country, Patient Preference and Adherence, 2015 July : 9 1017-1021

7. Thomson CJ, Berkowitz RG. Extradural frontal abscess complicating nasal septal abscess in a child. Int J Paediatr Otolarngol. 1998: 45:183-186

8. Yusuf S.; Kirk A. Case report: Nasal Septal Abscess. Emergency Medicine. 2014 April; E 46(4):179181

\section{Figures}




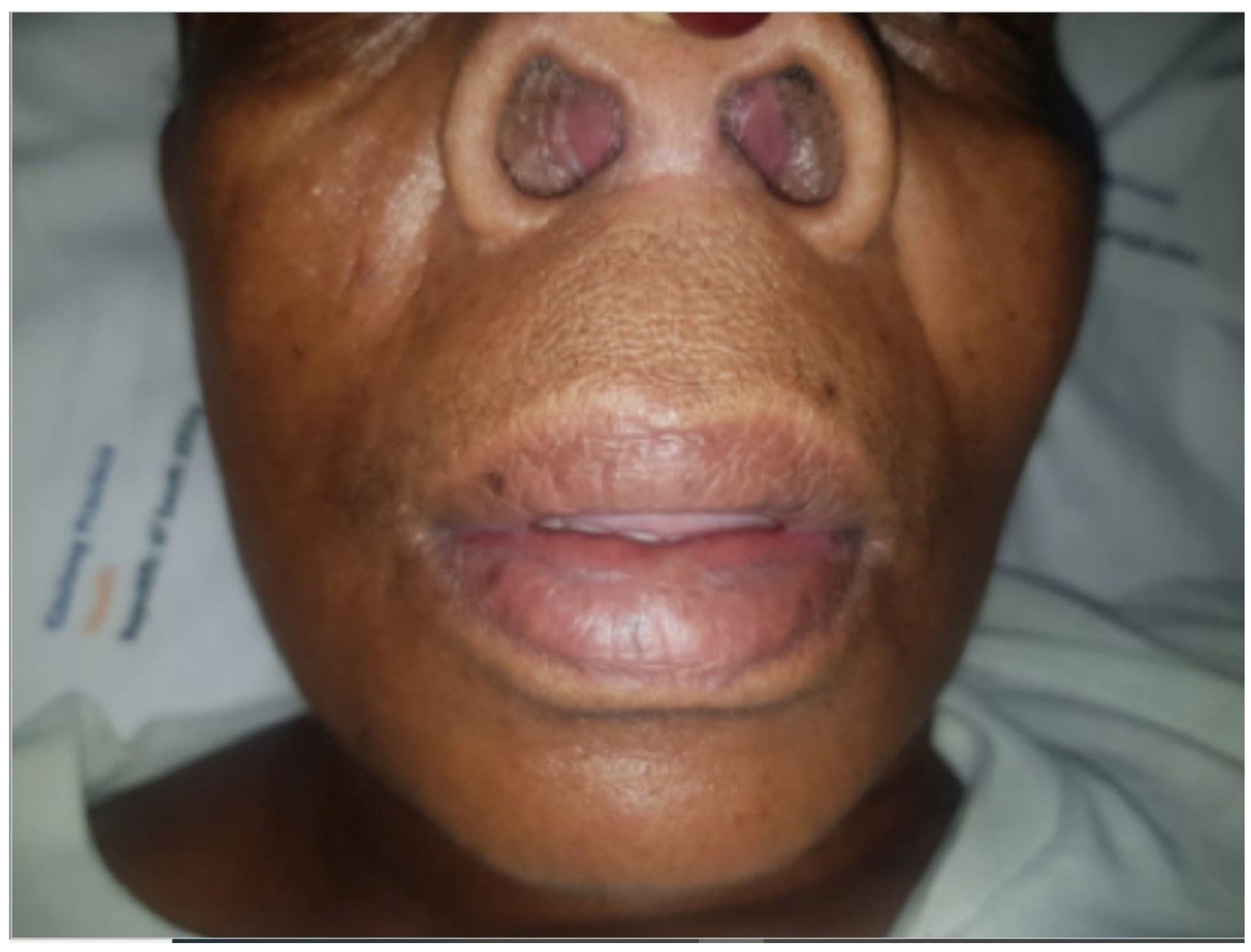

Figure 1

Nasal septal abscess occluding nasal passages 